\title{
Orion Entry, Descent, and Landing Performance and Mission Design
}

\author{
Joel M. Broome. ${ }^{1}$ \\ NASA/Johnson Space Center, Houston, TX 77058 \\ Dr. Wyatt Johnson ${ }^{2}$ \\ NASA/Johnson Space Center, Houston, TX 77058
}

The Orion Vehicle is the next spacecraft to take humans into space and will include missions to ISS as well as missions to the Moon. As part of that challenge, the vehicle will have to accommodate multiple mission design concepts, since return from Low Earth Orbit and return from the Moon can be quite different. Commonality between the different missions as it relates to vehicle systems, guidance capability, and operations concepts is the goal. Several unique mission design concepts include the specification of multiple land-based landing sites for a vehicle with closed-loop direct and skip entry guidance, followed by a parachute descent and landing attenuation system. This includes the ability of the vehicle to accurately target and land at a designated landing site, including site location aspects, landing site size, and landing opportunities assessments. Analyses associated with these mission design and flight performance challenges and constraints will be discussed as well as potential operational concepts to provide feasibility and/or mission commonality.

\section{Nomenclature}

$\begin{array}{lll}\text { AGL } & = & \text { Above Ground Level } \\ \text { ANTARES } & = & \text { Advanced NASA Technology Architecture for Exploration Studies } \\ \text { AOA } & = & \text { Angle of Attack } \\ C M & = & \text { Crew Module } \\ C O N U S & = & \text { Continental United States } \\ D O F & = & \text { Degree-of-Freedom } \\ E D L & = & \text { Entry, Descent and Landing } \\ E I & = & \text { Entry Interface } \\ F B C & = & \text { Forward Bay Cover } \\ G N \& C & = & \text { Guidance Navigation \& Control } \\ G P S & = & \text { Global Positioning System } \\ G R A M & = & \text { Global Reference Atmosphere Model } \\ I S S & = & \text { International Space Station } \\ L / D & = & \text { Lift-to-Drag ratio } \\ L E O & = & \text { Low Earth Orbit } \\ M S L & = & \text { Mean Sea Level } \\ n m i & = & \text { nautical miles } \\ R C S & = & \text { Reaction Control System } \\ R R A & = & \text { Range Reference Atmospheres } \\ S M & = & \text { Service Module } \\ T E I & = & \text { Trans-Earth Injection }\end{array}$

\footnotetext{
${ }_{1}^{1}$ Aerospace Engineer, Flight Mechanics and Trajectory Design, 2101 NASA Parkway / EG5

${ }^{2}$ Aerospace Engineer, Flight Mechanics and Trajectory Design, 2101 NASA Parkway / EG5, AIAA member
} 


\section{Introduction}

$\mathrm{T}$ He Orion vehicle is the next United States vehicle to take humans into Low Earth Orbit (LEO) and back to the Moon. The overall mission design aspects associated with return from the moon have been investigated in order to understand the requirements that need to be placed on the Entry, Descent, and Landing (EDL) portion of the mission. The strategy for returning the vehicle back from the moon was driven by the concept of return to a landlanding site in the Continental United States (CONUS) for all potential Earth-Moon orbital geometries. Another design consideration this strategy must accommodate is the disposal of the Service Module for a mission to Low Earth Orbit and returns from the Moon. The Service Module debris footprint must always be placed in the ocean 25 nmi from U.S. land masses and $200 \mathrm{nmi}$ from foreign land masses. The lunar return strategy was developed to understand the feasibility of commonality between ISS return and Lunar return landing sites. This strategy was also developed to provide a concept of operations for landing site selection associated with weather issues at designated landing sites and the performance capabilities of the Orion entry vehicle.

The Orion vehicle also has a requirement to land within a $5 \mathrm{~km}$ radius of the intended CONUS landing target. The analysis and concept development associated with meeting that requirement makes up the second portion of this paper. The analysis is broken up into two major sections, with and without knowledge of the day of landing winds. If day of landing winds is assumed, the concept of biasing the drogue deploy target to account for the winds of the day is analyzed to understand the advantage of adopting such a concept. This analysis was developed as a study to understand the feasibility associated with a $5 \mathrm{~km}$ landing accuracy requirement and to develop the parachute deployment strategy and operations concepts that must be employed to meet the requirement.

\section{Orion Lunar Return Strategy}

One of the goals of lunar return for the Orion vehicle is to provide lunar return capability to a landing site in the Continental United States (CONUS). In order to fulfill that goal, the Orion vehicle must accommodate all potential Earth-Moon geometries. In particular, the Entry, Descent, and Landing phase of flight must be able to execute long range entries on the order of $5400 \mathrm{nmi}$. The extent of the entry range is due to the Earth-Moon geometry which could place the antipode point well in the Southern hemisphere, which correlates to the Entry Interface (EI) location, but the CONUS landing target is in the Northern hemisphere. The antipode location could be anywhere between +28.6 and -28.6 degrees latitude, when the Moon is at maximum inclination in the metonic cycle. The Moon will travel from maximum declination (minimum antipode latitude), to minimum declination (maximum antipode latitude) in approximately 14 days, or half the lunar orbit. The associated EI point will be uprange of the antipode point, meaning that the entry interface point will be farther

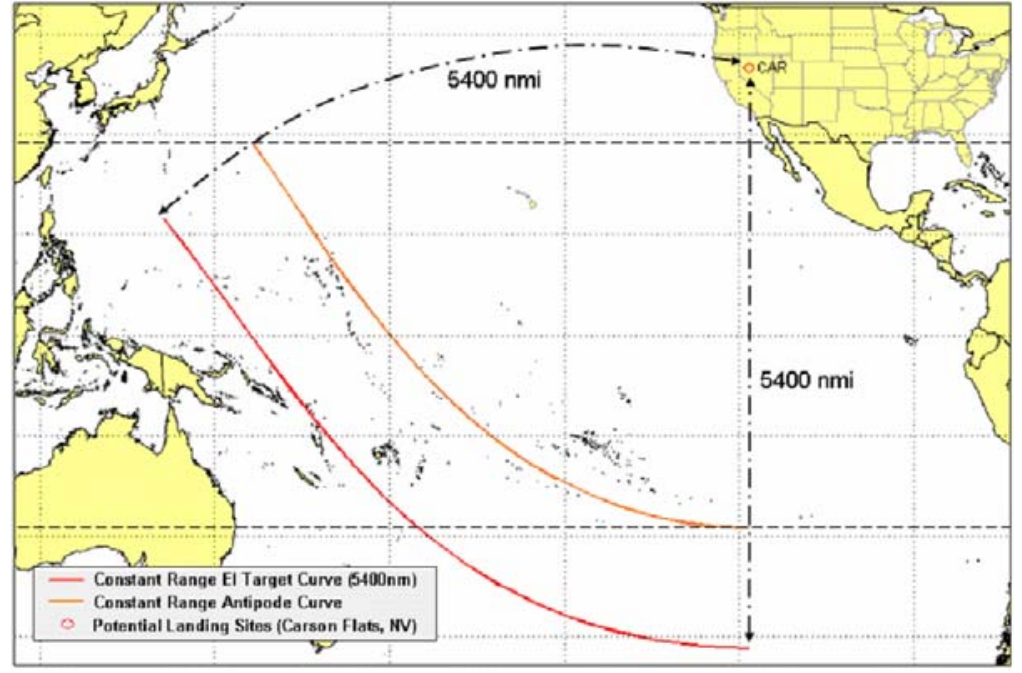

Figure 1. Constant Entry Range Approach (5400 nmi). away from the landing site than the antipode point. Initially, the goal was to be able to implement a constant range entry, which equates to a fairly consistent entry trajectory for all potential return approaches from the Moon. The longest entry range required is at the minimum antipode of -28.6 degrees. Assuming that range ( $5400 \mathrm{nmi}$ for Carson Flats, Nevada) is the same range that should be flown for all entries, an arc can be drawn showing all possible entry interface points. This proves to be a very simple approach and can be seen in Fig. 1. 
However, this approach doesn't take into consideration the location of the SM disposal debris footprint, which will be jettisoned prior to entry. Range safety requirements state that the SM debris must be kept at least $25 \mathrm{nmi}$ off U.S. territories and at least $200 \mathrm{nmi}$ off of foreign territories. Boundaries to support those requirements were developed by JSC/Mission Operations personnel and can be seen in Fig. 2, highlighted in cyan. Also included is a boundary line that defines the Eastern edge of international water ownership in the Pacific, which was taken from a world map. It is unknown at this time which boundary the Orion project will have to protect, so both are shown and utilized. The figure also shows the locations of the SM debris footprints and those colored in red do not meet the range safety requirements mentioned above.

The Trans-Earth Injection (TEI) maneuver, which is what will place the Orion vehicle on a trajectory to intersect the Earth, is equipped with

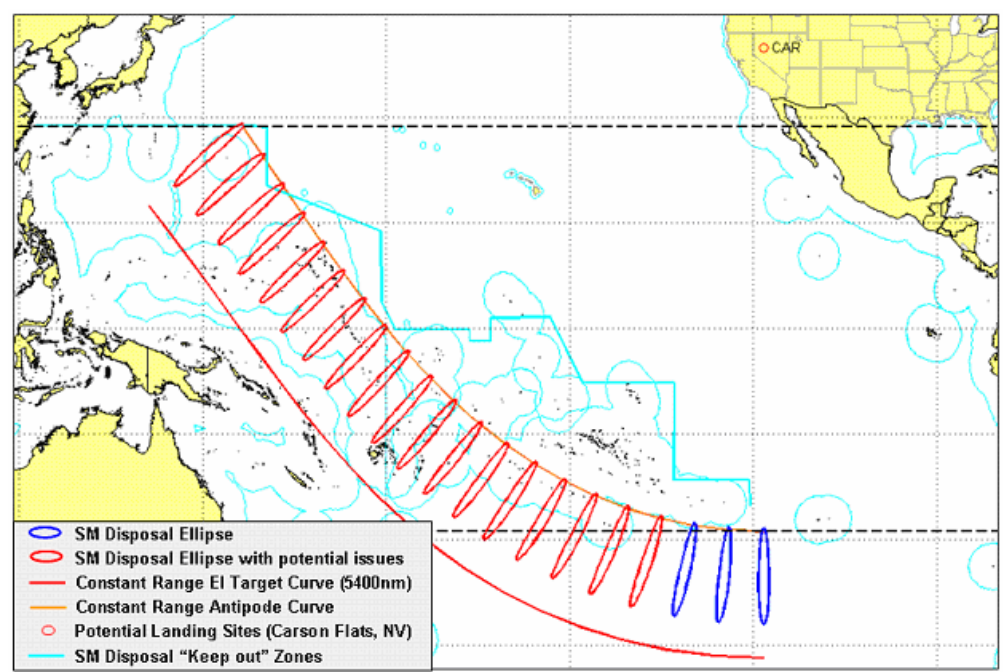

Figure 2. Constant Range Approach with SM disposal locations and disposal "keep out" zones.

enough performance to provide at least one-day variation in Moon-to-Earth flight time, which allows the earth to rotate a full 360 degrees of longitude. The TEI maneuver also has capability to adjust the Earth return approach direction by rotating the entry interface azimuth angle. These two capabilities allow unlimited movement of the entry interface point in the longitude direction. Even though the latitude of the entry interface point cannot be significantly modified, the movement in longitude and azimuth can be used to allow for SM disposal within the range safety requirements. Utilizing this concept, a new approach was formulated that requires the skip entry guidance to fly entry ranges from $\sim 3100 \mathrm{nmi}$ to $\sim 5400 \mathrm{nmi}$. For example, if the Earth-Moon geometry dictates an EI latitude near the equator, the EI longitude can be shifted East and the azimuth made more northerly to avoid SM disposal on Pacific islands. Figure 3 illustrates this concept.

The development of the lunar return strategy must also include the methodology to provide weather alternate landing sites and assessments on the vehicle performance required. Once the final burn in the TEI sequence has been initiated, the vehicle is on a course to intercept the

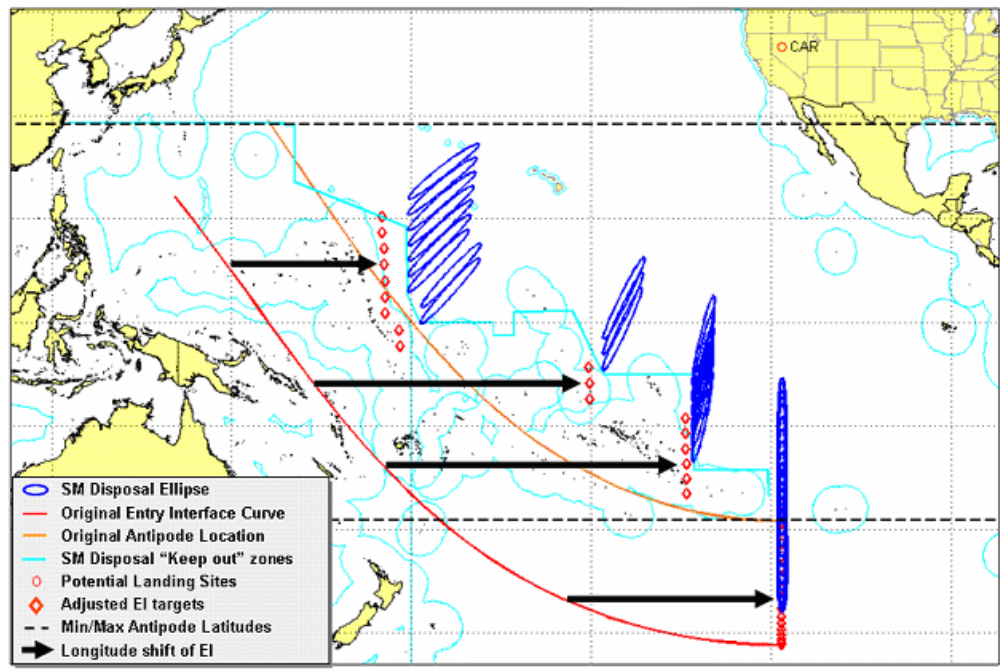

Figure 3. Demonstration of longitude and azimuth control concept to alleviate SM disposal issues.

Earth and there is no option to loiter

for weather issues to subside, which is currently the ISS return strategy. However, one option, specific to lunar return, is to use the skip entry guidance range and crossrange capability to fly to multiple landing sites, all from the same entry interface point. This would require an entry interface point that is not optimized for a given landing site, but rather a set of landing sites. This approach allows weather decisions to be made only hours prior to entry, rather than trusting 3-4 day weather forecasts or requiring a late propulsive maneuver to alter the entry interface conditions. A propulsive maneuver only 6-12 hours prior to EI will likely require significant delta- $\mathrm{V}$, but analysis is required to quantify the significance. The timing of such a maneuver and performance required would have to be 
weighed against the time interval acceptable for a weather forecast. So, utilizing the skip guidance capability seemed like the most attractive option and was further investigated. As mentioned previously, a skip guidance capability footprint was generated as part of the skip guidance development process. The skip capability footprint was developed by the Orion skip entry guidance team for varying $\mathrm{L} / \mathrm{D}$ vehicles. The footprint used in this analysis assumed a $0.4 \mathrm{~L} / \mathrm{D}$ vehicle. The capability footprint was overlaid for all possible entry interface conditions associated with the minimum to maximum antipode location possibilities. Since the crossrange capability of the vehicle increases with downrange, the EI locations were purposely chosen as far west as possible to maximize the crossrange. Each EI condition was targeted to a single point on the Earth's surface in hopes of enclosing as many landing sites as possible. Figure 4 illustrates this approach.

Again, this approach assumes a single target point was selected at the time of the TEI maneuver. This means that all landing sites that fall within the area defined by the overlap of the

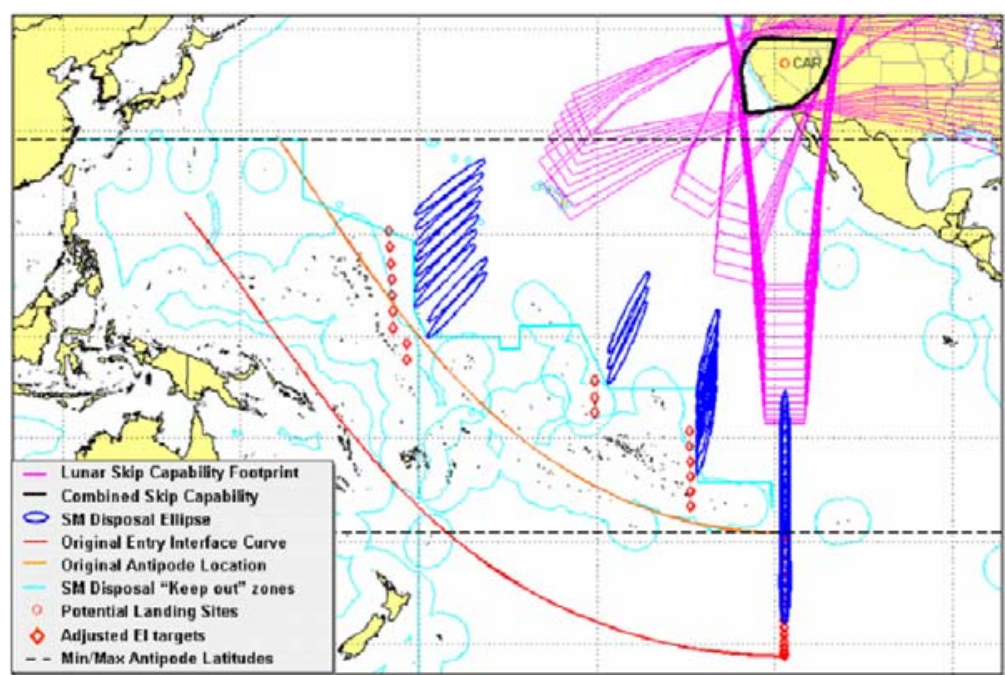

Figure 4. Overlay of skip entry guidance capability from most western entry interface locations. capability footprints (outlined in black) can be achieved from any lunar return approach throughout the Earth-Moon month. The single target point chosen at EI for a specific mission will be a location that optimizes the capability to achieve the official landing site network, which is not known at this time. Figure 5 shows the resulting area for a TEI maneuver targeted at Carson Flats, along with other potential landing sites. Figure 5 also shows the maximum in-land flight distance for an ISS return associated with the vehicle $\mathrm{L} / \mathrm{D}$ of 0.4. The area to the west of the restriction lines indicate areas the vehicle can achieve for ISS missions. Those ISS return restrictions assume no propulsive maneuver after SM separation by neither the SM nor the $\mathrm{CM}$. The separation distance between the SM debris field and the landing site achievable by the $\mathrm{CM}$ comes only from the difference in ballistic coefficients of the SM debris and the $\mathrm{CM}$ and the lifting capability of the $\mathrm{CM}$. Also plotted on figure 5 are the in-land distance restrictions associated with a $1000 \mathrm{nmi}$ separation distance between the SM debris field and the CM landing location. That in-land distance is arbitrary and would require a propulsive maneuver to achieve the separation distance. The shaded area designates the area where full commonality can be achieved for both

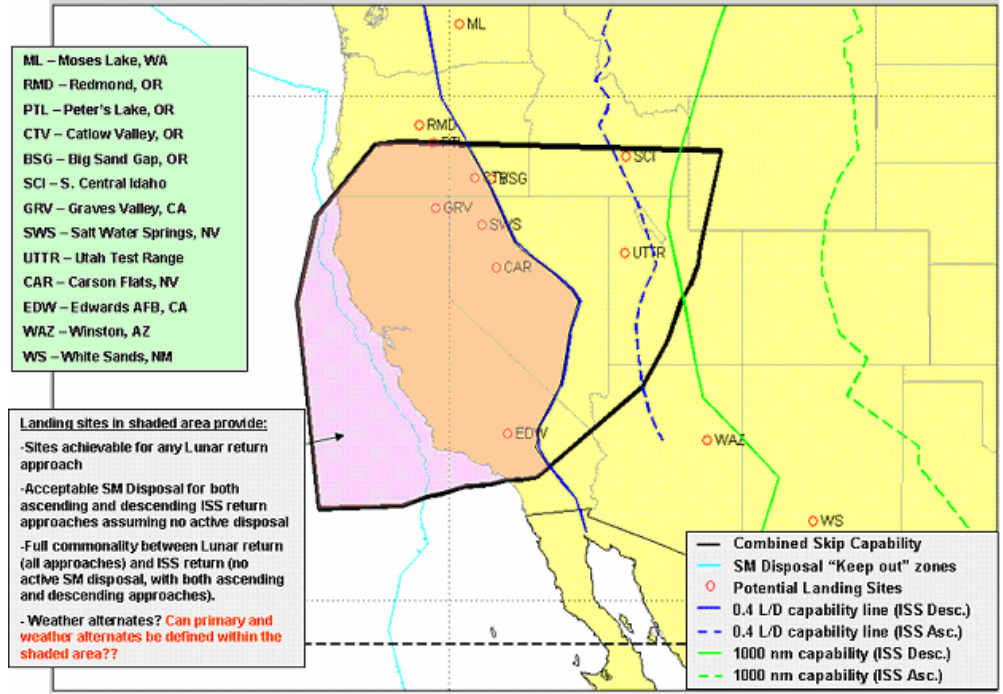

Figure 5. Overlay of Lunar skip capability from all EI locations and ISS return ranging capability. This plot demonstrates the potential for landing site commonality between the two missions.

Lunar and ISS return. The shaded region will account for the following: no active SM disposal for ISS return, both ascending and descending approaches available, single target location for TEI maneuver, and weather alternate capability for lunar returns. 
As suggested by Fig. 5, the question becomes one of weather independence within the shaded areas. Can both primary and weather alternates be defined within the shaded region? Preliminary analysis showed that North-South separation should provide the greatest probability for having one of two or three sites with acceptable weather conditions, indicating that two sites like Edwards AFB and Carson Flats or Edwards AFB and Graves Valley, CA would provide a good probability of weather independence. The analysis also indicated that East-West separation for a potential third landing site would further increase the probability of having acceptable weather conditions. This analysis indicates that for optimal weather independence a triangle shaped network may be ideal, suggesting Edwards, Graves Valley, and UTTR may be the best possible landing site network for weather independence while optimizing the commonality between the ISS and Lunar return missions. However, this is very preliminary and determining the appropriate landing site network was not part of this analysis and will require much more sitespecific analysis.

Overall, the analysis has shown that commonality between ISS return and Lunar skip return landing sites is achievable that accommodates all potential lunar return and ISS return latitudes. Commonality requires skip entry guidance to accommodate variable range entries from $\sim 3100 \mathrm{nmi}$ to $\sim 5400 \mathrm{nmi}$. This also assumes a non-active SM disposal and a vehicle $\mathrm{L} / \mathrm{D}$ of 0.4 . A reduction in $\mathrm{L} / \mathrm{D}$ would have a significant effect on this analysis by shrinking the skip capability footprint as well as the in-land distance achievable without active SM disposal for ISS missions. This could still be a viable solution at a reduced $\mathrm{L} / \mathrm{D}$, but would need further analysis. If commonality is not a goal or a variable range skip is not achievable, a near constant range skip entry that covers all entry latitudes could be attainable with sites in the Eastern United States.

\section{Orion Landing Accuracy Performance}

This section explains the analysis conducted to determine the landing accuracy capability of the Orion vehicle. The analysis was conducted in order to determine the appropriate value to place in the Constellation Architecture Requirements Document (CARD) for landing accuracy. The current landing accuracy requirement states that the vehicle must land within $5 \mathrm{~km}(2.7 \mathrm{nmi})$ of the intended CONUS target.

The major contributors to the landing accuracy footprint are the entry delivery accuracy to the drogue deploy target, the altitude variation at the drogue deploy target, and the drift under both the drogue and main parachutes due to winds. Analysis has shown that the primary footprint dispersion is due to the winds at the landing site and the subsequent vehicle drift, since there is no ability to control the vehicle state while under the parachutes. The analysis in this report focused on reducing the drift under the parachutes by modifying the drogue and main chute deployment triggers.

The analysis was divided into two major parts which were both investigated through Monte Carlo trajectory analysis. The first set of analysis assumes there is no day-of-landing wind knowledge. In general, the signature of this approach is a small drogue deployment target miss, followed by a slightly larger main deploy target miss, followed by a much larger miss at touchdown. The vehicle is generally always moving away from the center of the landing zone. The primary method for reducing the landing footprint in this analysis was to optimize the drogue and main parachute deployment triggers. This analysis also uncovered the need for a second trigger to accommodate the subsonic instability and deploy the chutes before the vehicle becomes completely unstable. The results of this analysis show that the $2.7 \mathrm{nmi}(5 \mathrm{~km})$ requirement would need to be increased by $1-2 \mathrm{~km}$ to provide adequate margins and achieve the 0.9973 reliability with $90 \%$ confidence.

The second set of analysis assumes that the day of landing winds can be measured 4-6 hours prior to landing. Those winds can then be used to offset the drogue deploy target. This approach provides the opposite signature as the no-wind knowledge case. The largest scatter from the center of the landing zone is at drogue deploy. At main deploy the vehicle is a little closer to the center of the landing zone, and at touchdown the footprint has reduced substantially and lies in the center of the landing zone. Utilizing this method, the landing accuracy requirement can be achieved at a 0.9973 reliability with $90 \%$ confidence and margin to spare.

Both sets of analysis show a strong correlation between the main deployment altitude and the landing footprint. As the main chute deployment altitude increases the landing footprint size increases. The main deployment altitude is driven by the minimum altitude needed to support the post-deployment activities before touchdown and the navigated altitude uncertainty. The main deployment altitude must be adjusted to account for the expected altitude uncertainty. As the uncertainty increases, the main deployment altitude must increase, also increasing the size of the landing footprint. Both sets of analysis show a strong dependence on navigated altitude accuracy in order to keep the landing footprint manageable. For the analysis with day-of-landing wind knowledge, GPS (+/- $100 \mathrm{ft})$ or near GPS quality (+/- 1000-2000 ft) accuracy in the navigated altitude is required to meet the $5 \mathrm{~km}(2.7 \mathrm{nmi})$ 
requirement. This can be accomplished by use of an alternate altitude measurement in the navigated altitude solution in the event of loss of GPS, or by guaranteeing the availability of GPS during entry (except the black-out zones of course).

\section{A. Constraints / Groundrules / Assumptions}

Constraints for both Analyses

1) The minimum altitude for main chute deployment was $5000 \mathrm{ft}$. This was to allow the minimum (no margin) timeline for reefing, heatshield deployment, and landing system configuration.

Assumptions for both Analyses

1) Only a single EI condition ( $4000 \mathrm{nmi}$, mid-range skip), and therefore a single skip entry trajectory was assumed. This assumes that the performance of the chute trigger is independent from entry trajectory associated with other approach azimuths and range. Any deviation in delivery accuracy to the drogue chute deploy point would be an issue with skip guidance, not with the chute trigger. Thus, all initial and final skip conditions were held constant as to not introduce extraneous noise in the results. However, it is unknown at this time if there is any coupling between landing accuracy and different skip profiles (e.g., altitude or Mach number at skip guidance termination as a function of entry range and azimuth).

2) The winds at Edwards Air Force Base were assumed to be the worst case for wind magnitude and the worst case for wind persistence of the 3 baselined landing sites.

3) Mass depletion during entry was modeled as an instantaneous mass subtraction at discrete events. At drogue deploy mass was subtracted to eliminate the RCS fuel, heatshield ablation, and Forward Bay Cover (FBC). Drogue mass was subtracted at main chute deploy and heatshield mass was subtracted at a navigated altitude of $4900 \mathrm{ft}$ AGL.

4) Analysis was conducted using the Numeric Skip Entry Guidance (NSEG) algorithm.

Assumptions specific to Wind Knowledge Analyses

1) The "balloon" data generated from GRAM 2007 were strictly vertical profiles and do not account for the drift of the balloon.

2) Small adjustments to the drogue deploy target will not significantly affect the trajectory flown by the entry guidance. This assumption allows the use of the range delta method described in section E.

\section{B. Simulation Environments}

All landing footprint analyses were conducted using the NASA-accredited 6-DOF simulation ANTARES. The simulation utilizes 6-DOF fidelity models in the areas of mass properties, RCS propulsion, and GN\&C algorithms and sensors. The simulation was used to model Monte Carlo dispersed trajectories involving both hardware and software dispersions, as well as vehicle and environment dispersions. The random seeds for each set of analyses were held constant, ensuring repeatability for the dispersions. Each Monte Carlo set simulated 3000 dispersed entry trajectories from EI to the ground. The list of parameters used in the Monte Carlo are listed in Fig. X.

The GRAM 99 atmosphere model is the baseline model for all atmospheric inputs. For analysis with no day-oflanding wind knowledge, the GRAM 99 atmosphere model was used to model the atmospheric conditions and winds from EI to the ground. Near the landing site, the GRAM 99 range reference database was used to model the atmospheric conditions and winds. The range reference database is based on actual measurements taken at the particular landing site and is more representative of the actual conditions.

When day-of-landing wind knowledge is assumed, the GRAM 99 atmosphere model was also used. However, the beta version of GRAM 2007 was used to generate the wind persistence data needed to assess the advantage of having wind knowledge. The GRAM 2007 model has the capability to model wind persistence for the desired delta time, or persistence time. In other words, GRAM 2007 will provide the winds at time zero, but can also predict what the winds will be like at any time in the future. The analysis investigated delta times of one, two, three, four, five, and six hours. The GRAM 2007 persistence data begins at 50,000 ft. In the period between 55,000 $\mathrm{ft}$ and $50,000 \mathrm{ft}$ the profile is linearly transitioned between GRAM 99 and the first point of the GRAM 2007 persistence data. This introduces a discontinuity because the GRAM 99 is for the current time and the persistence data represents a future time. A second approximation occurs due to the linear blending of the atmosphere profile; any short perturbations are lost during the blending.

\section{Analytical Techniques/Methodology}

The analysis was broken into two major tasks. The first was to assume there was no day-of-landing wind knowledge. Without wind knowledge, the task was to optimize the parachute sequencing and deployment triggers 
in an attempt to satisfy the current landing accuracy requirement. The second task was to assume winds of the day could be measured with a "balloon" launch at the landing site $X$ number of hours prior to landing. Assuming wind knowledge, the goal was to determine the appropriate drogue deployment and main deployment triggers that meet the current requirement, while maintaining acceptable deployment conditions. A secondary goal was to also optimize the triggers in order to minimize the landing footprint; however that analysis has not yet been completed.

For both sets of analyses, there are several ways in which the drogue chutes can be deployed. In general there is a deployment trigger that will deploy the chutes as downrange goes to zero if the vehicle is within an altitude box, and a deployment trigger based on Angle-Of-Attack (AOA) rate to counteract a subsonic stability problem. The actual drogue deployment scenarios are outlined below.

The drogues will be deployed if...

1) the vehicle has over flown the target, and goes below the upper bound of the altitude box

2) the downrange goes to zero and the vehicle is within the altitude box

3) the vehicle is still short of the target, but goes below the lower bound of the altitude box

4) the sensed AOA rate is greater than a specified rate limit.

For all analyses, the main parachute was deployed at an altitude above the ground level. The altitude selected was analyzed and had to be properly adjusted for the cases that simulated a degraded navigated altitude solution in order to preserve adequate timeline for post deployment vehicle configuration.

\section{Analysis Description: Without Day of Landing Wind Knowledge}

The purpose of this study was to design drogue and main parachute triggers that would allow the CM to land within a designated $5 \mathrm{~km}(2.7$ nmi.) radius footprint without using day-of-landing wind knowledge. The trigger must be able to tolerate fully dispersed EDL trajectories. After the chute is deployed, the CM drifts down to the ground with no further control (i.e., the chutes are not steerable). Since this scenario does not employ day-of-landing wind knowledge, the resulting wind drift can only serve to

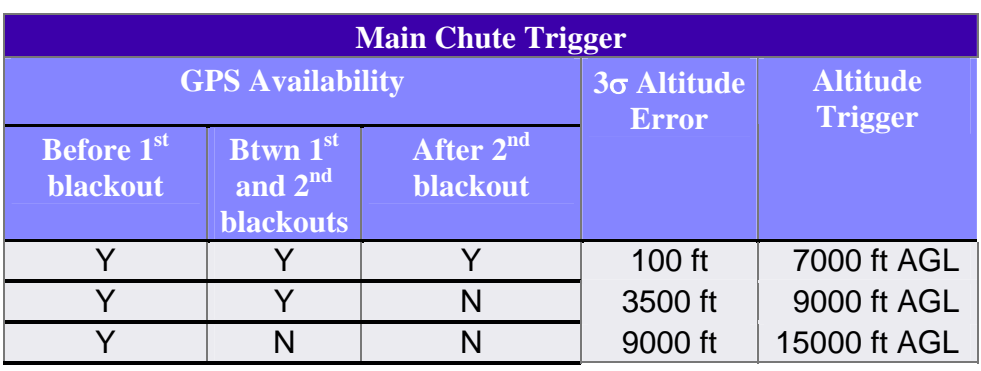

Figure 6. Altitude Uncertainty at main parachute deploy relative to GPS availability.

increase the footprint at touchdown relative to the footprint at drogue deployment. The only control over the landed footprint comes from a judicious choice of when to deploy the drogue chute, and when to deploy the main chute. The skip guidance algorithm guides the CM to a given latitude and longitude for drogue chute deployment. The altitude of guidance termination is not directly controllable.

According to the Range Reference Atmosphere (RRA) data for Edwards AFB, February is the worst-case month for winds in the drogue chute deployment range (35,000 to 45,000 feet MSL). Even though skip guidance may perfectly guide the vehicle over the target at 45,000 feet MSL, deploying the drogue chute while in the jet stream will result in more wind drift than had guidance flown past the target and out of the jet stream. Results show improved landing accuracy by sacrificing accuracy at drogue deployment for a lower chute deployment altitude. The strategy for deploying the drogue chute is to "deploy as low as possible". Under nominal conditions, this can be as low as 30,000 feet MSL.

The derived constraint preventing the drogue chute from being deployed lower than 30,000 feet MSL is the vehicle instability. The CM capsule becomes aerodynamically unstable below Mach 1, and tends to flip over and tumble. The RCS jets can stabilize the CM for a bit further. But as the vehicle slows down, and especially as the vehicle encounters any strong wind shear during this time period, the RCS system will not be capable of maintaining stability. For a single string RCS system, this occurs at around 35,000 feet MSL. If all 3 strings were used (for the final descent; altitude $<60,000$ feet MSL), most Monte Carlo cases could safely make it down to 20,000 feet MSL. 
Before the vehicle flips, there is a warning sign of excessive RCS control in the form of high angle of attack rates. As a precaution, an angle of attack rate trigger was added to the skip guidance to detect and prevent flips from happening by deploying the drogue chute early. By deploying early, the vehicle is saved from a fatal flip, but will be exposed to additional wind drift, which could potentially increase the overall landing footprint.

The remaining choice is on the main chute deployment. Again, to minimize wind drift, deployment should be as low as possible. 7,000 feet AGL was selected as a starting point (with 5,000 feet as the absolute minimum as stated

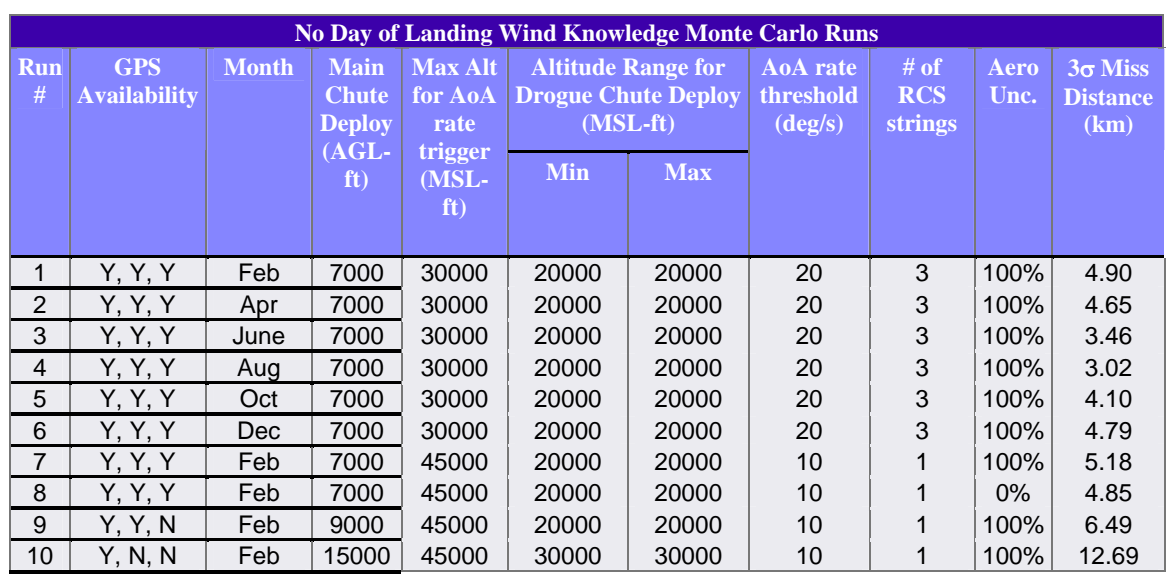

Figure 7. No day of landing wind knowledge run matrix.

earlier), balancing perceived risk versus gain. The 3-sigma navigated altitude error is added to this to produce the final altitude trigger. If GPS measurements are available during this phase, the 3 -sigma altitude error is negligible, at about \pm 100 feet. Figure 6 provides the set of main parachute altitude triggers as a function of GPS availability. Figure 7 provides the run matrix used for this analysis with the corresponding 3-sigma miss distance for each scenario.

\section{E. Analysis Description: With Day of Landing Wind Knowledge (Balloon Data)}

Using the GRAM 2007 capability to produce both winds at time zero as well as the wind persistence at given time intervals, 3,000 atmospheric profiles were generated for time zero (T0). Each 3,000 set had 250 profiles per month. Also, 3,000 atmospheric profiles were generated at each time interval from 1-6 hours after time zero, in one hour increments in order to simulate the wind persistence expected over that time interval. The 3,000 atmospheric profiles at time zero were run within the simulation using nominal conditions. Use of nominal conditions is intended to represent the procedure that would be used operationally when a balloon is launched prior to entry. The touchdown points for each of these cases were recorded. The T0 through $\mathrm{T}+6$ hour wind profiles

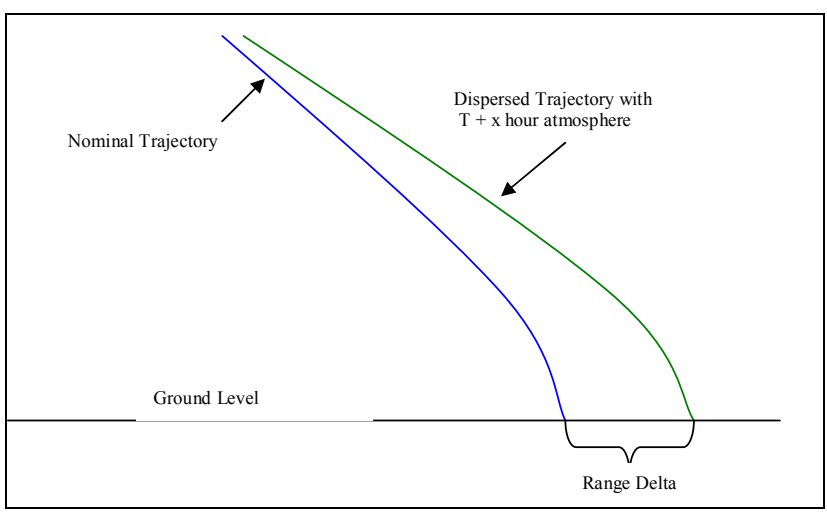

Figure 8. Illustration of range delta method.

were then run

using dispersed

entry conditions.

The touchdown

points from the

Monte Carlo

simulations were

subtracted from

the nominal

touchdown points,

and the resulting

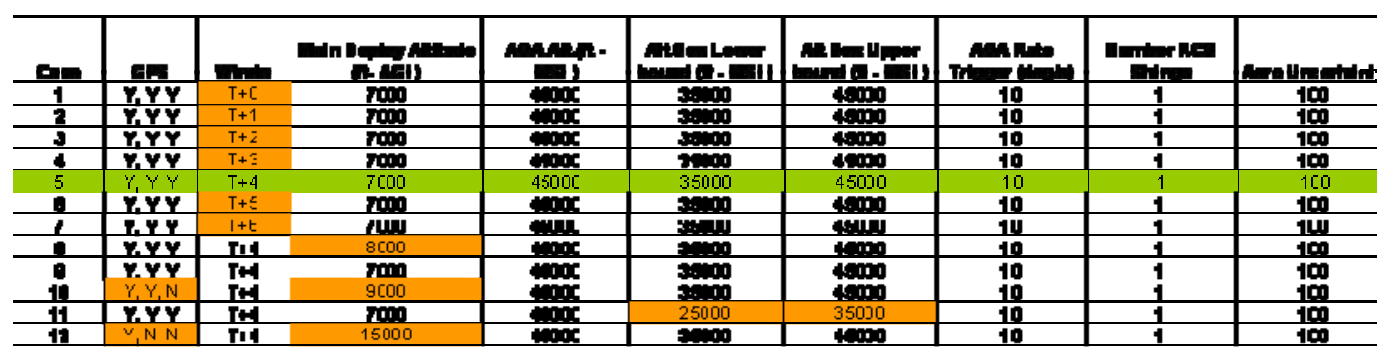

Figure 9. With day of landing wind knowledge run matrix.

range delta was

recorded as the "miss" distance. For the operational scenario, the actual approach would be to take the result of nominal simulation and then adjust the drogue deploy target for the actual entry. The range delta method that is 
used here makes the assumption that adjusting the drogue deploy target will not affect the trajectory that the entry guidance commands; most importantly that the drogue deploy altitude will not change. An illustration of the range delta method is shown in Fig. 8.

Additional parametric analyses were performed to determine the sensitivities to drogue and main chute deployment altitude, GPS availability, and vehicle mass. The $T+4$ hour atmospheric profiles were used as the baseline for the parametric analyses. Estimates of operational capabilities indicate that processing balloon data four hours prior to touchdown is an achievable task. The full run matrix is enclosed in Fig. 9 with the baseline case highlighted in green and the variations in orange. Case nine, which has no color variation, was the same as the baseline but assumed a lighter vehicle at drogue deploy by $1000 \mathrm{lbs}$.

\section{Landing Accuracy Analysis Results}

\section{A. Without Day-of-Landing Wind Knowledge}

Ten cases were considered in assessing landing accuracy using monthly mean winds (no day-of-landing wind measurements) to bias the nominal guidance target. These cases are summarized in Fig. 7. Runs 1-6 are meant to depict the performance relative to time of year using the most optimistic assumptions - GPS is fully available from entry to touchdown (except during the blackout phases), and with all three RCS strings used to control attitude during the final descent. With all three RCS strings in use, the vehicle remains stabilized during the descent (even while passing through the jet stream with its associated high wind shears). The vehicle is able to descend to an altitude of 20,000 ft MSL and deploy the drogue chute. The drogues inflate, and 20-35 seconds later, the vehicle reaches $7000 \mathrm{ft}$ AGL, and the main chutes are deployed. Three to six minutes after that, the CM touches down.

The last four runs characterize performance under more likely scenarios. Run seven is analogous to Run one, but with only one RCS string used. With less protection against flips, the angle of attack rate trigger is enabled at a much higher altitude and with a lower threshold. As a result, many of the cases within Run seven had to deploy higher and thus drifted farther, resulting in a larger footprint.

Run eight examined the influence of the uncertainties in the dynamic derivatives in the subsonic regime (specifically the Cmq and $\mathrm{Cnr}$ ) to the

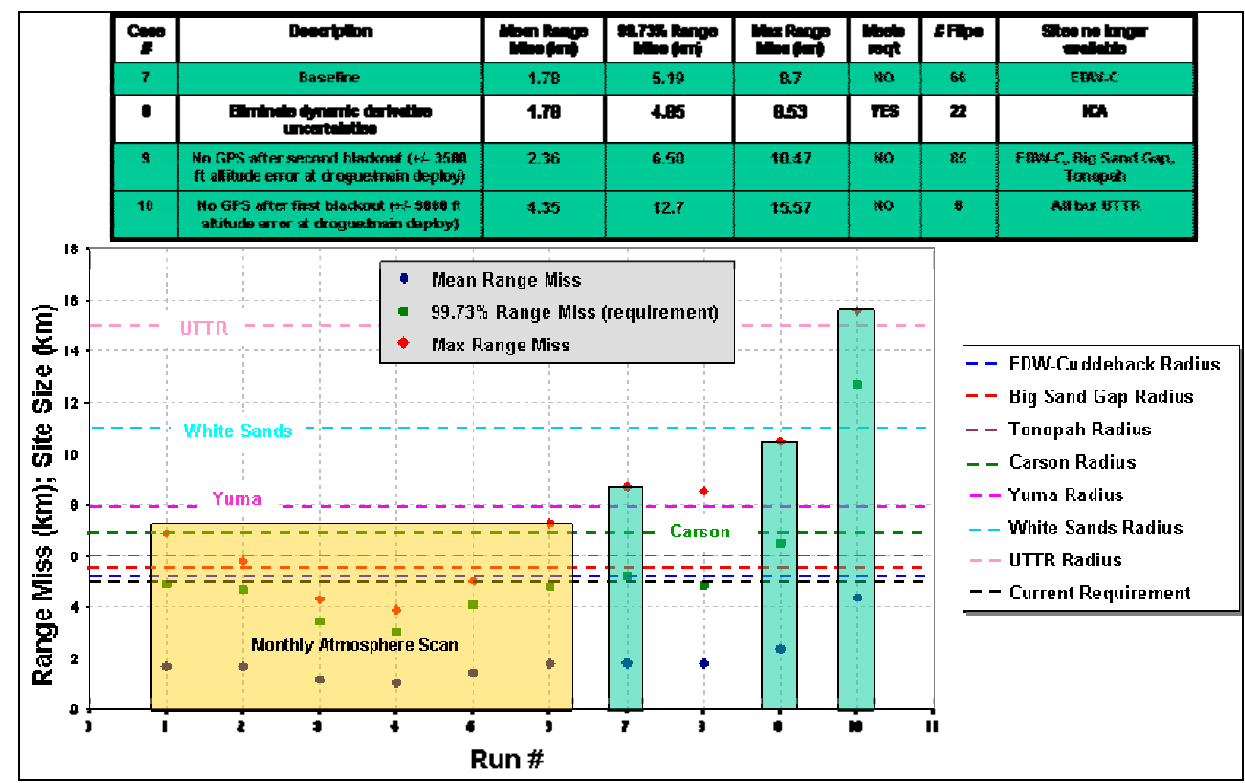

Figure 10. Monte Carlo landing footprint size (radius) assuming no day of landing wind knowledge and how it relates to the size of several potential landing sites.

likelihood of the vehicle flipping over (or equivalently, causing the angle of attack rate trigger to force deployment at a higher-than-desired altitude). While many cases were saved by removing the aerodynamic uncertainties, most of the flip-cases are caused by excessive wind-shear, and were thus unaffected.

The last two runs, Run nine and Run ten, looked at the sensitivity of altitude uncertainty on landed footprint performance. With a degraded navigation solution, these cases are forced to deploy the drogue and main chutes at a correspondingly higher altitude to preserve the timeline. At the time of this analysis, a barometric altimeter measurement was not available for comparison. Preliminary work suggests that it could achieve an altitude uncertainty of $+/-1000$ feet to 2000 feet.

Based on this analysis, the landing accuracy requirement would have to be increased to accommodate the landing footprint capability. It may be possible to achieve the $5 \mathrm{~km}$ landing accuracy without day-of-landing wind 
knowledge, but GPS or a similar altitude measurement would need to be guaranteed and the subsonic instability issue would need to be addressed. Figure 10 provides the Monte Carlo landing footprint data for each run plotted alongside several potential landing sites. As the landing footprint increases, it becomes impossible to place a landing circle at some of the landing sites. Increasing the landing accuracy requirement would eliminate certain sites as options for Orion landing sites.

\section{B. With Day-of Landing-Wind Knowledge (Balloon Data)}

The results utilizing day of landing wind knowledge are presented in two sections. The first represents the baseline data. The raw range errors (before the range delta method was applied) for the nominal scenarios and the Monte Carlo scenarios for the $\mathrm{T}+0$ hour through $\mathrm{T}+6$ hour data were generated first. The results indicate that all cases (even the nominal) violate the 5 $\mathrm{km} \quad(2.7 \mathrm{nmi})$ range requirement without wind knowledge. Once the range
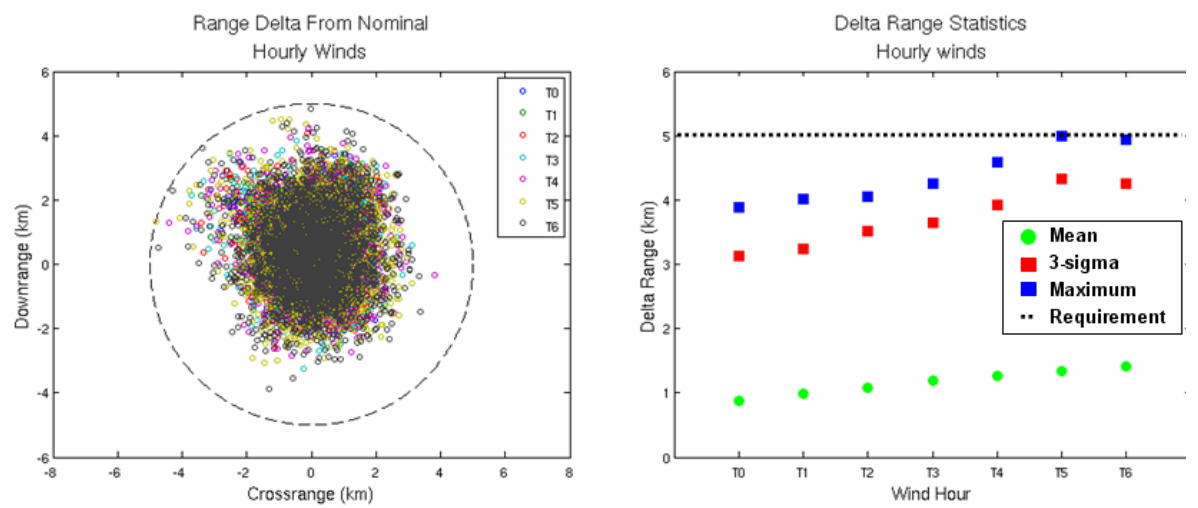

Figure 11. Touchdown errors and statistics after range delta method is applied. This shows the benefit of using the winds of the day to offset the drogue deploy target.

delta method was applied, the miss distance drops to within the $5 \mathrm{~km}(2.7 \mathrm{nmi})$ range requirement as shown in Fig. 11 for up to six hours of wind persistence.

Examination of the statistics, also in Fig. 11, shows the expected trend that the uncorrected range error increases as the "staleness" of the balloon data increases, except from the time +5 hour to time +6 hour persistence. There is no definitive explanation for the perceived anomaly where the $\mathrm{T}+5$ hour data has a higher maximum and a higher $99.73 \%$ value. Potential causes are the small sample size (only 250 cases per month) or a flaw in the GRAM 2007 model.

The baseline results have shown that day of landing wind knowledge is a highly deisrable tool for meeting the $5 \mathrm{~km}(2.7 \mathrm{nmi})$ touchdown accuracy requirement. The parametric analyses are designed to investigate sensitivities to key parameters and test the limits of the range delta method. Note that the parametric cases in Fig 12 are denoted by scenario numbers 1-5, which correspond to cases 8-12 in the full run matrix in Fig. 9. All the parametric cases require a re-run of the nominal entry trajectory Monte Carlo set due to the changes to the nominal parachute sequence or input

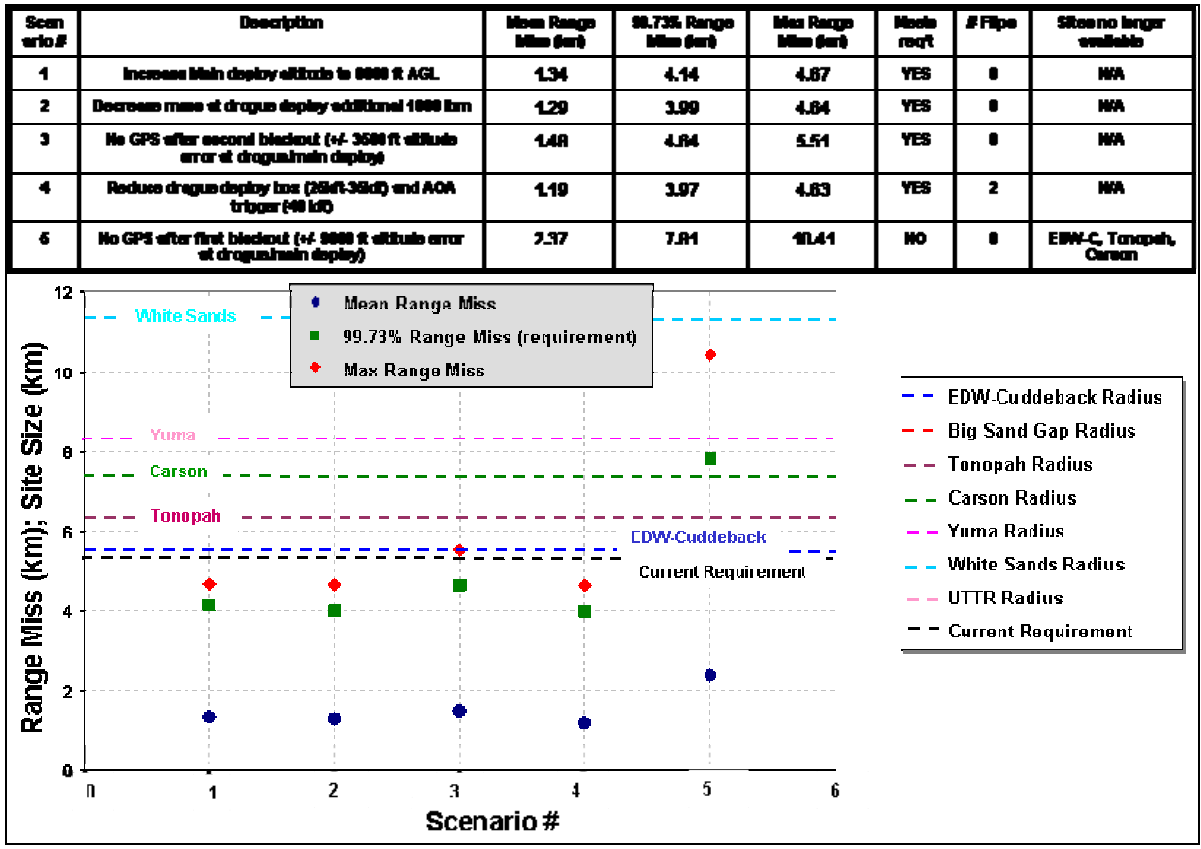

Figure 12. Monte Carlo landing footprint size (radius) with day of landing wind knowledge and how it relates to several potential landing sites. All scenarios except for \#5 meet the 3-sigma requirement of $5 \mathrm{~km}$.

10

American Institute of Aeronautics and Astronautics 
parameters. These are required in order to complete the range delta method of calculating the target miss.

Lowering the mass at drogue deploy by $1,000 \mathrm{lbm}$ (scenario \#2) and lowering the drogue deployment box by $10,000 \mathrm{ft}$ (scenario \#4) had minimal effect on the landing accuracy footprint and still meet the requirement. The remaining scenarios $(\# 1, \# 3, \# 5$ ), increased the main chute deployment altitude to $8,000 \mathrm{ft}, 9,000 \mathrm{ft}$, and 15,000 ft respectively. Scenarios \#3 and \#5 also varied the GPS availability; which is what forced the main parachute deployment higher in altitude. This must be done to account for the increase in altitude uncertainty associated with the loss of GPS availability, but still ensure mains are deployed high enough to provide adequate timeline for vehicle configuration before touchdown. The increase in the altitude uncertainty and subsequently the main parachute deployment altitude increases the size of the landing footprint and violates the requirement for scenario \#5. Figure 12 shows the landing footprint results for each of the five scenarios and provides the landing footprint relationship to the size to several potential landing sites.

\section{Orion Landing Accuracy Conclusions}

In order to meet the $5 \mathrm{~km}(2.7 \mathrm{nmi})$ Orion landing requirement, or reduce the landing requirement, day of landing wind knowledge is required. Based on the current analysis, winds must be measured at least six hours prior to landing. The two key recommendations to the Orion project from this analysis are the requirement for accurate navigated altitude and the requirement to measure day of landing winds 4-6 hours prior to landing. The requirement for altitude accuracy will have some impact to the vehicle design by either increasing the criticality of GPS or requiring alternate altitude measurement instrumentation. Obtaining the day-of-landing wind knowledge will place requirements on ground and mission operations to measure winds of the day, process the winds, and provide an offset to the drogue deploy target that must be up-linked to the vehicle.

Without wind knowledge, the $5 \mathrm{~km}(2.7 \mathrm{nmi})$ landing requirement would need to be increased $1-2 \mathrm{~km}$ to provide adequate margins and achieve the 0.9973 reliability with $90 \%$ confidence. However, as the landing accuracy requirement increases above $5 \mathrm{~km}(2.7 \mathrm{nmi})$, some landing sites are no longer available because they are not large enough. As a note, the only reason to reduce the landing accuracy requirement, from a landing site selection perspective, would be to allow the Roger's Lakebed at Edwards to be a potential landing site (needs to be reduced from $5 \mathrm{~km}$ to $4 \mathrm{~km}$ ), or to increase the overall number of potential landing locations.

One of the most significant parameters affecting touchdown accuracy is the deployment altitude of the main chutes. The $5 \mathrm{~km}(2.7 \mathrm{nmi})$ accuracy requirement was not achievable, even with wind knowledge, when the main chute deployment was raised to $15,000 \mathrm{ft}$ AGL. The minimum altitude of main deployment is determined by the timeline constraints associated with heatshield jettison and landing system configuration, as well as the navigated altitude uncertainty. As the uncertainty grows, the main chute deployment altitude must increase to preserve the minimum altitude required for post-deployment vehicle configuration activities prior to landing. With the current design of the parachute deployment triggers, accurate navigated altitude is required to meet the landing accuracy requirement of $5 \mathrm{~km}(2.7 \mathrm{nmi})$. This analysis shows it is possible to achieve that altitude accuracy using GPS assets, however, this would require GPS acquisition after the initial plasma blackout during entry. In the case where GPS is not available after the initial plasma blackout, preliminary analysis also shows that the navigated altitude would be greatly improved using an alternate altitude measurement, possibly a barometer, as part of the navigated altitude solution. One of those two solutions, or something equivalent, must be included to meet the current requirement. Further analysis will also investigate alternate parachute triggers that could reduce the sensitivity to navigated altitude. At a minimum, that analysis will include parachute triggers based on navigated velocity and drag acceleration.

\section{Acknowledgments}

This sections needs to be updated

Skip Guidance Team.

ANTARES Simulation development team

\section{References}

This sections needs to be updated.

Skip guidance paper.

Entry Nav Paper

ANTARES development paper. 
American Institute of Aeronautics and Astronautics 